\title{
An Innovative Risk Assessment Tool for Prospective Risk Analysis to Improve the Quality in Health Care: The Bow-Tie
}

\author{
Haitham Shoman* and Samer Ellahham \\ Sheikh Khalifa Medical City (SKMC) Managed by Cleveland Clinic, Abu Dhabi, UAE and Cleveland Clinic Foundation, Ohio, USA
}

Received: January 17, 2018; Accepted: February 05, 2018; Published: March 05, 2018

*Corresponding author: Haitham Shoman, SKMC Managed by Cleveland Clinic, Abu Dhabi, UAE and Cleveland Clinic Foundation, Ohio, USA, E-mail: haitham.sh89@gmail.com

\begin{abstract}
In order to improve the quality of health care, it is very important to assess the risks that might affect the service delivery and quality of health intervention. This will help other health care professionals to work on ensuring minimum risks are maintained in delivery a certain intervention to the patients. There are several models used in the the health care industry that helps in conducting a risk assessment, but there have been a gap in demonstrating a user-friendly model for the health care professionals. The Bow-tie model is a prospective risk assessment tool that can be conducted by several health care professionals to assess the potential risks in a certain department or intervention and offers a pictorial graphical presentation for easy understanding for all staff.
\end{abstract}

Keywords: Risk assessment; Bow-tie; Quality; Health care;

\section{Introduction / Background}

In healthcare, serious adverse events and medical errors can easily occur jeopardizing patient safety and continuity of treatment [1]. However, studies have revealed that identifying risks and working on analyzing and mitigating them, have proven to lower down undesirable and preventable healthcare associated events [2]. The Health and Safety Executive defines hazard as " anything that may cause harm, such as chemicals, electricity, working from ladders, an open drawer etc." and risk as "the chance, high or low, that somebody could be harmed by these and other hazards, together with an indication of how serious the harm could be" [3]. In healthcare, there are several events that could be prevented such as falls in hospitals, surgical site infections, drugs' side effects and post-operative complications and having a risk assessment model in place would help deployment of barriers to prevent the occurrence of these events.

In order to improve patient safety and ultimately the quality of healthcare, one should systematically analyze and assess the risks for healthcare errors, and determine their possible causes. Reducing and preventing morbidity and mortality in healthcare is of paramount importance in the medical profession and in healthcare politics [4]. There are a few risk analysis models like the Health-Care Failure Mode And Effect Analysis (HFMEA), Root Cause Analysis (RCA), the Swiss Cheese model and the Bow-tie model [5-7]. In this study the Bow-tie risk analysis model is being explored due its extensive use in several industries and its ability to provide a pictorial presentation of the risk assessment process and potentially being the lead healthcare risk analysis tool.

The history of the Bow-tie extends to 1979 where diagrams were drawn in the Imperial Chemistry Industry course notes at the University of Queensland, Australia but the exact origin is not yet clears [8]. After the catastrophic Piper Alpha platform incident in 1988, Lord Cullen mentioned the critical importance of having systematic approach in identifying, assessing and controlling hazards [8]. The Bow-tie was then adopted by the Royal Dutch / Shell group in the early nineties as a standardized methodology for risk assessment and control across their world sites [8].

Industries such as the oil and gas, aviation, nuclear, transport and petrochemical are full of hazards and healthcare is no less. The Bow-tie model was originally developed to analyze hazard and accidents in high risk industries as such in Europe and Australia; it is being used to conduct risk assessment and to prevent hazards [9]. Since 2010, as with many risk assessment tools, it has been adopted by many industries and it is spreading widely in other fields where the healthcare sector is one of them [9-12]. Following the extensive use of Proactive Risk Assessment (PRA) in hazardous industries, there has been a growing need for having PRA methods that can identify, eliminate or prevent contributing factors leading to adverse and hazard events $[13,14]$. Despite the existence of several PRA, only a limited number has been applied and not widely used [15]. The Bowtie model is a risk analysis tool that prospectively analyses and visualizes risks, causes and consequences of potential hazards and adverse events. In healthcare, the Bow-tie methodology is a barrier analytical tool that systematically identifies, prioritizes and controls clinical risks that can threat patient safety [1]. It brings together causes, errors, preventive and recovery measures and consequences, and observes the significance and causes of current safety risks. Thus, it prospectively prioritizes riskminimizing interventions illustrating the relationship between 
causes and consequences of undesirable events in a simple comprehensible way and visualizing shortcomings and gaps as unsuccessful or missing safeguards and barriers [16]. The Bowtie methodology incorporates both prospective and retrospective approaches offering users the convenience to understand the situations before and after the incidence of an event [1]. A review of the literature shows that the Bow-tie model as a risk assessment tool in healthcare has only been used in a very limited number of studies and proved to be effective $[1,4,16]$. This article discusses the Bow-tie model showing a pictorial presentation of its application in risk assessment and how would it fit in the healthcare sector.

\section{Discussion}

The bow-tie methodology includes a risk management cycle that covers hazard identification, risk evaluation and risk control along with adverse events that may occur [17]. It commences by identifying the major event which is the centre of the diagram [17]. On the left-hand side are the threats that can trigger the major event in the centre. On the right-hand side of the diagram (right to the major event in the centre) lie the consequences. After identifying the major event, threats and consequences, barriers can then be identified [16]. Barriers can be preventive or protective [1]. Preventive barriers are those that already exist in the threats' path to prevent their initiation and protective barriers can protect or minimize the adverse events caused by threats [1]. Sometimes the barriers could be jeopardized by an escalation factor. Barriers that are identified following the emergence of escalation factors are called secondary barriers. A comprehensive
Bow-Tie diagram would have a pictorial presentation of the threats, consequences, escalation factors and the barriers along the pathways [4]. Recommendations and strategies could then be proposed based on the preventive and protective barriers.

Hazards can be identified from existing reports, events, conferences and team members' input. Studies have shown that deploying adequate risk analysis methodologies in healthcare operations are effective in reducing harm and undesirable events to healthcare beneficiaries $[1,4,16]$. The Bow-Tie diagrams are also useful in presenting the relationship between the individual, organizational and cultural factors that contribute in the way risks are managed [1]. It is important to have a model that shows all barriers in an eye-friendly manner where the user can easily navigate easily through and look at all the threats in one place rather than having to flip over pages and sometimes lose track of the threats and what needs to be done. Non-healthcare industries that have plenty of risks associated with the nature of their work have been using the Bow-Tie and their staff have all contributed to its development in analyzing risk where some people have even referred to it being an goal oriented model to improve the quality of a service and mitigate risks effectively [17]. In this model, error detection and recovery is easily presented offering staff the insight of their role in different phases of the recovery process including identifying, preventing and fixing errors.

In addition, the three studies that used the Bow-Tie methodology in risk assessment, have shown that it was successful in promoting healthcare staff awareness about threats and errors and errors provoking conditions in their environments ad workplace.

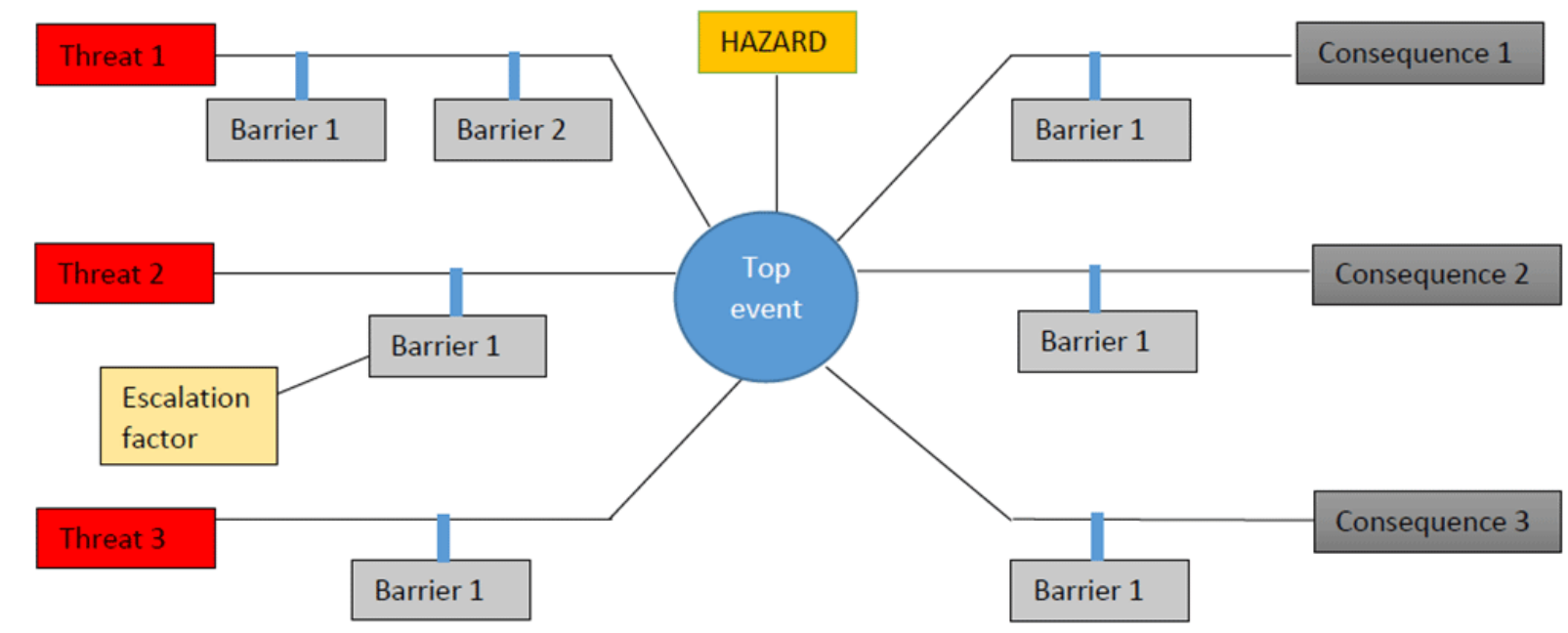

Figure 1: The bow-tie diagram

Despite the usefulness, practicality and great potential of this tool, it has its limitations. The Bow-Tie can be time-consuming in its development requiring extensive work and awareness on how to use it $[1,4,16]$. The practicality and reliability of the outputs depends on the inputs given. Data gathering needs to be adequately managed and should be of high quality for reliable results $[1,4,16]$. Since it is considered a new model in the healthcare industry, users of the model needs to be trained on how to use it along with the necessary software for its implementation with particular emphasis on the terminologies of threats, consequences, hazards, escalation factors, hazards and barriers $[1,4,16]$. Sometimes in a model, the results generated cannot be generalized across all healthcare settings especially when the inputs used were of explicit nature for a specialized healthcare setting $[1,4,16]$. 


\section{Bow-tie terminologies [17]}

It is important to understand the terminologies and its definitions while conducting a bow-tie analysis:

- Hazard: Anything related to the topic of interest with a potential to cause harm to safety, health, environment or reputation.

- Threat: A direct and independent potential cause that can lead to the hazard being released by producing the top event leading to a consequence.

- Top event: This is when the hazard is released; it is considered the first event in a series of negative events causing undesired consequences

- Control: Any action taken that can act against some undesired efforts in order to maintain a desired situation; Proactive controls (on the left side of the bow-tie diagram) prevent events from occurring and reactive controls (on the right side of the bow-tie diagram) minimize consequences.

- Escalation factor: A condition that jeopardizes the effectiveness of a control leading to increased risk.

- Consequence: The undesired event is a result of a released hazard that leads to loss or damage in safety, persons, environments, assets or reputation.

\section{Conclusion}

The Bow-Tie has been used in several industries as a comprehensive risk assessment model. Health care is also an industry where risks are always found. A few studies has been conducted so far to show how this model was effective in prospective risk assessment and mitigation of hazards to ensure high quality of health care service delivery to the patients. More studies are needed to explore the clinical effectiveness of this model in several health care settings.

\section{References}

1. Abdi Z, Ravaghi H, Abbasi M, Delgoshaei B ES. International Journal of Health Care Quality Assurance Article information . 2016.

2. Oliver D, Daly F, Martin FC, McMurdo MET. Risk factors and risk assessment tools for falls in hospital in-patients: A systematic review. Age Ageing. 2004;33(2):122-130.

3. Health and Safety Executive. Risk assessment. 2017. Available from: http://www.hse.gov.uk/risk/controlling-risks.htm
4. Kerckhoffs MC, van der Sluijs AF, Binnekade JM, Dongelmans DA. Improving Patient Safety in the ICU by Prospective Identification of Missing Safety Barriers Using the Bow-Tie Prospective Risk Analysis Model. J Patient Saf. 2013;9(3):154-159. doi: 10.1097/ PTS.0b013e318288a476.

5. Prous J. The changing face of healthcare. EBR - Eur Biopharm Rev. 2009;31(WINTER):66-67.

6. JT R. Understanding adverse events: Human factors. Clin risk Manag. 1995;31-54.

7. Zegers M, de Bruijne MC, de Keizer B, Merten H, Groenewegen PP, van der Wal G, et al. The incidence, root-causes, and outcomes of adverse events in surgical units: implication for potential prevention strategies. Patient Saf Surg. 2011;5(1):13.

8. CGE: Risk Management Solutions. The History of Bowtie. 2017. Available from: http://www.cgerisk.com/knowledge-base/riskassessment/the-bowtie-methodology

9. Jacinto C, Silva C. A semi-quantitative assessment of occupational risks using bow-tie representation. Saf Sci. 2010;48(8):973-979.

10. Hudson P. Applying the lessons of high risk industries to health care. Qual Saf Health Care. 2003;12(Suppl 1):i7-i12. doi: 10.1136/qhc.12. suppl_1.i7

11. FAA FAA. Safety Risk Management Guidance for System Acquisitions ( SRMGSA ) Federal Aviation Administration Safety Management System ( SMS ) and Acquisition Management System ( AMS ) Guidance Document. 2008

12. De Dianous V, Fiévez C. ARAMIS project: A more explicit demonstration of risk control through the use of bow-tie diagrams and the evaluation of safety barrier performance. J Hazard Mater. 2006;130(3):220-233.

13. Clancy CM. Patient Safety in the Intensive Care Unit. Patient Saf Intensive Care Unit. 2014;3(1):1- 5.

14. Vincent C. Understanding and Responding to Adverse Events. N Engl J Med. 2003;348(11):1051-1056.

15. Lyons M. Towards a framework to select techniques for error prediction: Supporting novice users in the healthcare sector. Appl Ergon . 2009;40(3):379-395.

16. Wierenga PC, Lie-a-huen L, Rooij SE De, Klazinga NS, Guchelaar H, Smorenburg SM. Application of the Bow-Tie Model in Medication Safety Risk Analysis: Consecutive Experience in Two Hospitals in the Netherlands. Drug Saf. 2009;32(8):663-673. doi: 10.2165/00002018200932080-00005

17. Saud YE, Israni KC, Goddard J, Americas ERM, Practice R, Ten P, et al. Bow-Tie Diagrams in Downstream Hazard Identification and Risk Assessment. Process Saf Prog. 2014;33(1):26-35. 